\title{
Conceptos y percepciones de las abuelas sobre el cuidado de sus nietos. Estudio cualitativo en una población del Hospital Italiano de Buenos Aires Grandmothers, concepts and perceptions in caring for their grandchildren. Qualitative study in a population from the Italian Hospital of Buenos Aires
}

\author{
Dra. Mariela A. Weisbrot ${ }^{a}$ y Dra. Nanci Giraudo ${ }^{a}$
}

\section{RESUMEN}

En las familias actuales, los abuelos son fuente importante de cuidado de nietos. La bibliografía mundial es contradictoria respecto de si el cuidar nietos afecta en forma negativa, positiva o no afecta la salud de los abuelos. El objetivo del presente estudio es explorar los conceptos y percepciones de las abuelas, pertenecientes al Plan de Salud del Hospital Italiano de Buenos Aires, sobre el cuidado de los nietos.

Métodos. Estudio exploratorio, descriptivo con técnicas cualitativas. Se realizaron dos grupos focales con abuelas de entre 50 y 75 años, cuidadoras de nietos al menos $9 \mathrm{~h}$ a la semana. Se llegó a la saturación del discurso. A partir de la información obtenida se realizó la transcripción, el análisis y triangulación de los datos.

Resultados. Las abuelas maternas son las más convocadas. El motivo principal es ayudar a la familia mientras los padres de los niños trabajan. El ser abuelas cuidadoras parece ser un deber implícito, un pensamiento instituido y se plantea a la familia como la mejor proveedora de cuidados. La mayoría coincidió que cuidar nietos constituye un acto muy gratificante y placentero. Las abuelas que destinan más horas al cuidado de sus nietos o las que conviven con ellos presentan mayor estrés, reclamos por falta de reconocimiento, sensación de sobrecarga y agotamientos, además de peor salud percibida. Conclusión. El cuidado delos nietos proporciona satisfacción, sensación de utilidad y dinamismo $\mathrm{y}$, como aspectos negativos puedegenerar estrés, falta de tiempo personal, trastornos en la salud y conflicto con los padres.

Palabras clave: abuelas, cuidadores, nietos.

a. Hospital Italiano de Buenos Aires, Servicio de Medicina Familiar y Comunitaria.

Correspondencia:

Dra. Mariela A.

Weisbrot:

mariela.weisbrot@

hospitalitaliano.org.ar

Conflicto de intereses: Ninguno que declarar.

Recibido: 21-10-2011 Aceptado: 23-1-2012

\section{SUMMARY}

Grandparents are an important source in caring of grandchildren. In the world literature there are several contradictions regarding the positive, negative or no effects on the health of the grandparents by taking care of grandchildren. The aim of this study is to explore the concepts and perceptions of grandmothers, belonging to an insured population from the Italian Hospital of Buenos Aires, while caring for their grandchildren.

Method. Exploratory and descriptive study that used qualitative techniques for compiling the information. We made two focal groups with grandmothers aged 50-75 years old, who take care of their grandchildren at least 9 hours per week. Altogether 13 women took part, achieving the saturation of the speech. Transcription, analysis and triangulation of information was carried out from obtained data.

Results. Maternal grandmothers are the most called. The purpose is to help the family while parents are at work. Being grandmother caregiver seems to be an implicit duty and an established thought, where the family is considered the best way of taking care of children. The majority agreed that taking care of a grandchild constitutes a very rewarding and even pleasant act, although it was also observed that those who spent more day hours taking care of their grandchildren or that were living together with them, experiment stress, claims for lack of recognition, sensation of overcharge and depletions, and reported a worse health condition.

Conclusion. Caring for grandchildren provides satisfaction, feelings of vitality and dynamism. On the negative side, can lead to stress, lack of personal time, health problems and conflict with parents.

Key words: grandmother, caregivers, grandchildren.

http:/ /dx.doi.org/10.5546/aap.2012.126

\section{INTRODUCCIÓN}

Los abuelos son fuente importante de cuidado de nietos. En EE.UU. y en Madrid 14,5\% y 70\% de las abuelas respectivamente cuidaron un nieto por 6 meses o más. ${ }^{1-3}$

Las abuelas maternas son las más convocadas y el motivo es proveer sostén afectivo o económico a sus hijos. ${ }^{4-6}$

La mayoría de los estudios que analizan la repercusión del cuidado de nietos se limita a abuelos cuyos hijos están ausentes por motivos graves y experimentan depresión, insomnio, hipertensión arterial, diabetes, limitaciones en la funcionalidad y empobrecimiento de la salud comunicada. ${ }^{7-10}$

La bibliografía muestra contradicciones en cuanto a la repercusión so- 
bre la salud de los abuelos que cuidan a sus nietos tiempo parcial. ${ }^{11}$ Hay trabajos que lo relacionan con mayores problemas de salud, ${ }^{7-9,12-17}$ pero otros muestran que la salud mejora. ${ }^{18}$ La cantidad de horas dedicadas pareciera tener relación directa en cuanto a la peor salud comunicada. . $^{5-7}$

La concepción de salud/enfermedad es una construcción sociocultural. Existe un complejo mundo de creencias y valores, normas, conocimientos y comportamientos ligados a la salud que dan cuenta de la relación que el sujeto tiene con su cuerpo, con los alimentos, con los hábitos de vida y con el tiempo libre, entre otros. ${ }^{19,20} \mathrm{La}$ posibilidad de percibir un problema de salud está condicionada por factores como la responsabilidad o el rol familiar que la persona desempeñe y las prioridades cotidianas. ${ }^{21}$

El objetivo fue explorar los conceptos y percepciones de las abuelas del Plan de Salud del Hospital Italiano de Buenos Aires (PS HIBA) sobre el cuidado de los nietos.

Los objetivos específicos fueron: conocer las razones por las que las abuelas cuidan a sus nietos; explorar percepciones, sentimientos y sensaciones respecto de lo que significa cuidar nietos; conocer las opiniones acerca de cómo afecta a la salud el hecho de cuidar nietos; indagar cómo incide el cuidado de los nietos en el manejo de su tiempo y explorar si el rol de abuela cuidadora influye en la relación con los padres de los nietos.

\section{MATERIAL Y MÉTODO}

Fecha: diciembre del año 2008 a junio del año 2009.

Diseño: estudio exploratorio, descriptivo, cualitativo y de corte transversal.

Población: abuelas de 50 a 75 años con nivel primario completo que cuidan o cuidaron al menos un nieto por lo menos 9 horas a la semana, durante al menos 4 meses consecutivos en los últimos 3 años.

Reclutamiento: se captaron las abuelas mediante el método de bola de nieve*. Solicitamos a 40 médicos del Servicio de Medicina Familiar del PS HIBA que identifiquen e inviten a participar del estudio a dos abuelas que cumplieran los criterios de inclusión. Se solicitaron los datos de las posibles participantes para verificar los criterios de inclusión y confirmar asistencia. Obtuvimos 25 participantes que cumplían criterios de inclusión, de las cuales asistieron 13.

Recolección de datos: se realizaron 2 grupos focales con 7 y 6 participantes respectivamente, a cargo de una coordinadora y una observadora llegando a saturar el discurso. ${ }^{22}$

Análisis de los datos: se realizó la trascripción de los discursos a un documento y se procedió al análisis temático utilizando el Nud-ist (QSR N6 For Windows 98, NT4, 2000, ME \& XP) según los tópicos definidos. El análisis se basó en la Teoría Fundamentada (TF), metodología general enraizada en información sistemáticamente recogida y analizada. ${ }^{23}$

Las dos investigadoras realizaron en forma independiente el pre-análisis del contenido, la identificación y categorización de los temas, la comparación de las áreas temáticas identificadas y la interpretación. ${ }^{24}$ A partir de la información obtenida, se realizó la transcripción y análisis mediante la técnica de triangulación entre los investigadores y algunos participantes en los grupos. ${ }^{25,26}$

Los discursos fueron grabados previo consentimiento verbal.

Protocolo $\mathrm{N}^{\mathrm{o}} 1227$ aprobado el 26 de junio de 2008 por el Comité de Ética de Protocolos de Investigación del Departamento de Docencia e Investigación del Hospital Italiano de Buenos Aires.

\section{RESULTADOS}

Las 13 participantes presentaban entre 54 y 75 años, con una edad promedio de 63 años. Once (11) participantes eran abuelas maternas, solo 3 trabajaban al momento del estudio y solo 3 convivían con sus nietos. Ocho (8) abuelas eran casadas y 5 viudas. Siete (7) abuelas cuidaban un nieto, 3 abuelas cuidaban dos nietos y 3 cuidaban tres nietos.

Del desglose de la información e identificación de los tópicos, los investigadores iniciaron la interpretación de la información (ver Figura 1).

Un mayor detalle de las respuestas obtenidas en los grupos focales (GF) se encuentra en forma de Anexo en versión electrónica.

\section{Razón del ser cuidadora}

Las abuelas pertenecen a familias donde ambos padres trabajan todo el día. La mayoría dijo asumir el cuidado de sus nietos por voluntad y porque sienten la obligación de ayudar a sus hijos,

\footnotetext{
* El método bola de nieve consiste en la captación de personas para participar en un grupo focal a partir de un referente en la comunidad donde se va a trabajar. Como requisito, las personas no deben conocerse entre sí, con el fin de facilitar la dinámica grupal.
} 
por cuestiones económicas y por confianza en la calidad del cuidado, alegando que la familia siempre es la mejor proveedora de cuidado.

Algunas dijeron colaborar económicamente con sus hijos ofreciendo su propia casa y, al vivir todos bajo un mismo techo, se dividen las funciones, siendo la abuela la que se encarga del cuidado de los nietos. Otras dijeron cuidar a sus nietos solo algunas horas.
[...] Yo la cuidé desde que nació. Mi hija alquilaba y se vino a vivir a casa para poder hacerse su casa. (GF 1 - Párrafo 2).

[...] El bebé tenía 1 año y medio, lo iban a llevar cerca de mi casa a una guardería, todo el día. Entonces, yo decidí cuidarlo algunas horas. (GF 2 - Párrafo 81).

Algunas abuelas cuidan a los nietos por un "deber ser", sintiéndose responsables de la crianza de los niños. Otras están solas y sin ocupación,
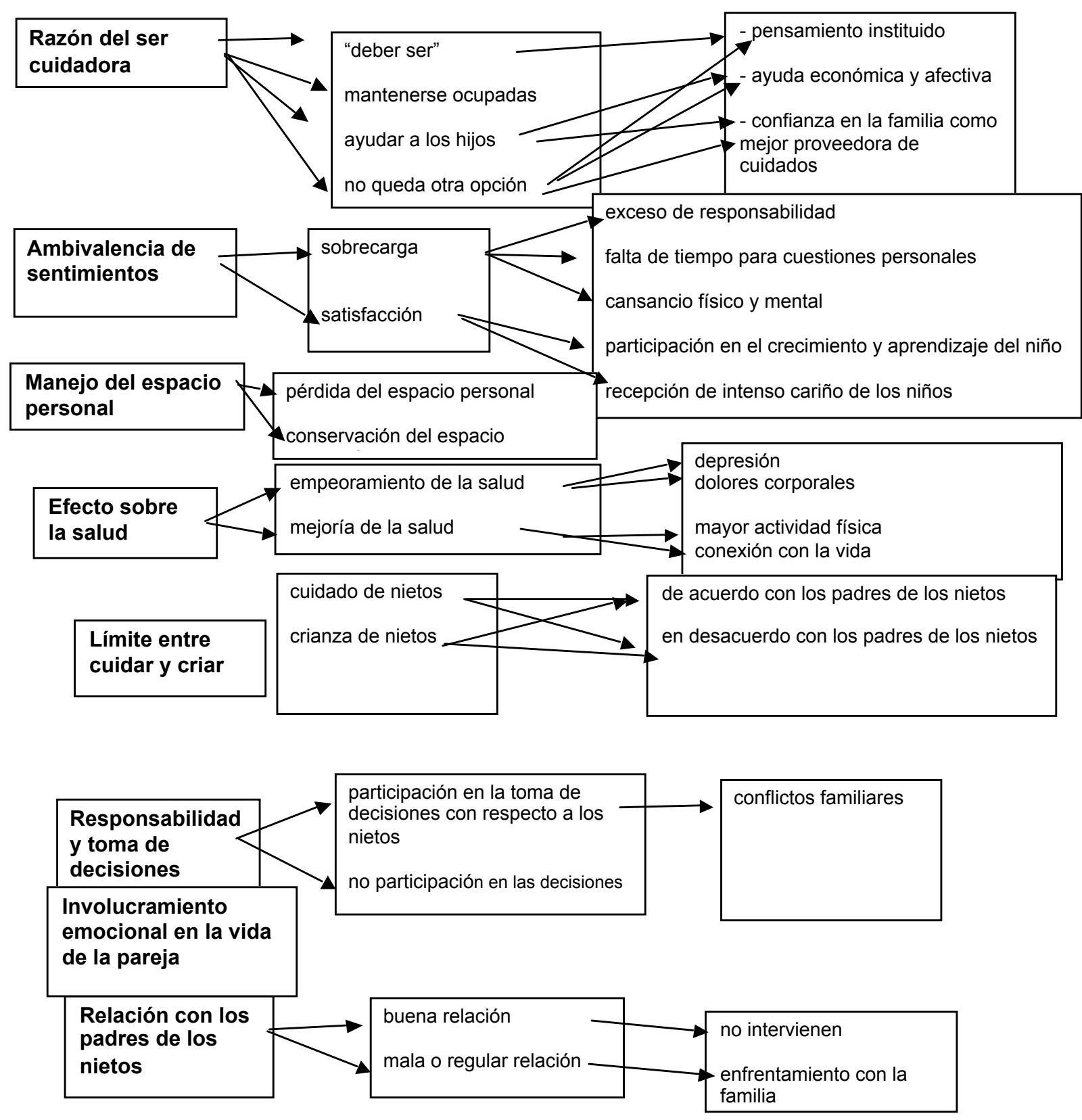
y recuperaron el placer de vivir cuidando a sus nietos porque consideran que es una forma de mantenerse ocupadas, útiles y queridas.

[...] Mi marido y yo vivíamos juntos y cuando me quedé sola... me pasé la vida en la casa de mi hija cuidando a mis nietos, solamente volvía para dormir. (GF 2- Párrafo 125).

\section{Ambivalencia de sentimientos}

Un aspecto que surgió es la ambivalencia de sentimientos que les genera el ser cuidadoras. Pasando de la satisfacción que les genera el cuidar los nietos al cansancio, sobrecarga y esfuerzo físico que les implica tal responsabilidad.

[...] Soy feliz porque te da tanto amor esa criatura, esa nena que me dice abu, cuéntame un cuento o vamos a armar el tablero con las letras. (GF 1- Párrafo 48).

[...] Yo creo que está relacionado con la cantidad de horas que lo cuidamos.; Qué felicidad cuando llegan y qué felicidad cuando se van! (GF 2- Párrafo 173).

Las responsabilidades, el trabajo y el cansancio son mayores para las abuelas que conviven con sus nietos.

[...] Otro tema es vivir con hijos donde no te puedes desligar. Yo cierro mi dormitorio y de golpe escucho la puerta de mi habitación y el ven abuela... (GF 1- Párrafo 18).

\section{Manejo del espacio personal}

No parece sencillo para algunas abuelas contar con un momento personal. En cambio otras, por decisión propia o de los hijos, sí pueden preservar un tiempo para ellas y cuidan a sus nietos algunas horas.

[...] Yo estaba tan atada como usted, que dejé de ir a mi médico. Iba cuando ya no daba más, cuando me dolía todo. Entonces empecé a decirle a mi hija, tal fecha tengo que ir al médico. Empieza a acomodarte con tu trabajo y con los niños. (GF 1- Párrafo 37).

\section{Efectos sobre la salud}

Algunas abuelas están muy a gusto con su condición de cuidadoras, se sienten muy bien de salud y en mejores condiciones físicas.

[...] En un principio me di cuenta de que me faltaba movimiento. En dos meses tomé ritmo. Con respecto a la salud yo creo que logré incluso adelgazar. Antes no tenía la misma vitalidad y el dinamismo que tengo ahora que cuido a mi nieta. (GF 2- Párrafo 187).

En el otro extremo se encuentran abuelas que refieren haberse enfermado de depresión, fibromialgia y dolores corporales a raíz de cuidar a los nietos.

[...] La angustia que tenía fue mejorando desde que la nena va al jardin, ahora estoy mucho mejor, es mucha la responsabilidad. (GF 2- Párrafo 122).

\section{Espacio en las decisiones de la crianza}

El cuidar a los nietos implica un gran esfuerzo para poner límites. La mayoría dijo ser más flexibles con los nietos que lo que fueron con sus propios hijos.

A pesar de la cantidad de horas, algunas abuelas dicen claramente que ellas cuidan a sus nietos y que los encargados de criarlos son los padres. Mientras que otras se involucran con la crianza y prefieren ponerse de acuerdo con los padres para no generar contradicciones en el niño.

[...] Dime cómo la quieres criar a la nena para poder saber hasta dónde decirle esto no, lo otro sí. (GF 1- Párrafo 20).

Algunas abuelas encuentran dificultoso poner límites sin contradecir o interferir con las decisiones de los padres. Otras, por momentos chocan con los padres a la hora de poner límites.

[...] ¿Hasta dónde llego?... A mí me cuesta, pero tengo que encontrar el límite para no pasarme del otro lado, respetarlos como núcleo familiar, no meterme, no opinar, no decidir. (GF 2- Párrafo 161).

\section{Toma de decisiones}

Hay situaciones con los nietos, como los problemas de salud, que parecen sobrepasar a la mayoría de las abuelas. Se sienten más inseguras para cargar con la responsabilidad.

[...] Se me termina el mundo cuando tiene algún problema como fiebre. Cómo puede ser, con mis hijos solucionaba el problema, no llamaba a nadie... pero con la nena la situación me pone nerviosa. (GF 1- Párrafos 49 y 50).

Otras, el pasar más horas con los nietos y la familia, les habilita un poder de decisión importante. Estas abuelas se sienten cómodas con la responsabilidad de decidir y hasta pueden ofenderse si no se les consulta o si no se les delega responsabilidades.

[...] Cuando uno llega al médico... "pero vino la abuela" y "dígale a la madre que me llame", es la respuesta del profesional. Yo entiendo que la mamá es la mamá, pero la abuela un poco se gana el lugar. Creo que uno cubre los agujeros que encuentra y no por elección. (GF 2- Párrafo 151).

\section{Involucramiento emocional}

Algunas abuelas que tuvieron depresión dijeron vivir de cerca problemas importantes de la pareja de padres sintiéndose muy afectadas emocionalmente. Dado que los niños sufren a partir 
de los problemas de pareja, las abuelas inmersas en ese ambiente, en general, hacen propio ese sufrimiento.

[...] Y a mí me hizo muy mal esa separación (llora). El nene quedó en casa con mi hijo y tuvo algunos problemas... pero yo me encargué de todo. (GF 2- Párrafo 82).

\section{Relación con los padres de los nietos}

Indagamos la relación de las abuelas con los padres de los niños, especialmente con la familia política, y en general dijeron tener una relación afable con ambos progenitores, solo una abuela tenía una relación conflictiva con su propia hija y con el yerno.

La mayoría tiene la filosofía de no enfrentarse con la familia política, de respetar las reglas que imponen en la casa. En general deciden "cerrar la boca" para tener una buena relación.

[...] Con mis hijas a veces puedo tener algún problemita, pero con los yernos, nunca. Porque yo siempre tengo la política de que ellos tienen razón. Me respetan mucho. (GF 1- Párrafo 76).

Si bien expresaron buena relación y la posibilidad de conversar sobre los desacuerdos con los padres sin problema, pareciera que el cuidar a los nietos les otorga un poder especial a la hora de discutir las diferencias.

\section{DISCUSIÓN}

En concordancia con otros estudios observamos que las abuelas provienen de familias donde ambos padres trabajan jornada completa y que decidieron cuidar a sus nietos para ayudar a sus hijos. Las abuelas maternas son las convocadas ${ }^{5,6}$ y a diferencia de la mayoría de los trabajos publicados, ninguna estuvo obligada por circunstancias críticas como ausencia de los padres. ${ }^{7-11}$ Varias familias dan por hecho esta forma de cuidado como un "rol natural y esperado". $5,27-29$

Percibimos dos formas de ayudar a los hijos. Una, ofreciendo su propio trabajo de cuidadora sin remuneración económica para que lo hijos puedan trabajar y ahorrar dinero, y la otra, porque los abuelos brindan cuidados de mejor calidad que las personas ajenas a la familia., ${ }^{3,50}$

La responsabilidad que les representa el cuidar a los nietos les genera un esfuerzo físico y mental importante. Como muestran otros estudios, ${ }^{3,5}$ muchas de estas abuelas, sobre todo las que conviven con los nietos o los cuidan mayor cantidad de horas, quedan agotadas sintiendo una sobrecarga muy importante. No disponen de tiempo para descansar, para realizar actividades placenteras ni cuidar su salud. Surgió la sensación de sentirse no saludables. Oscilan entre un estado de felicidad debido al amor entregado por los nietos, la alegría de verlos crecer y un estado de cansancio, estrés y sobrecarga. Vemos que el "deber ser" abuela cuidadora y ayudar a la familia es un mandato muy fuerte y que les resulta muy difícil cuestionar o dejar entrever sentimientos de hostilidad o de renuncia.

Como describen otros investigadores, ${ }^{14,28,30}$ pareciera ser que el hecho más relacionado con la falta de salud es el haber atravesado situaciones familiares conflictivas. Las abuelas que dijeron tener depresión, en general, se encontraron envueltas en situaciones familiares angustiantes, como la separación de los padres, enfermedad de los niños y exceso de control por parte de las madres de los niños.

Al igual que Grinstead ${ }^{12}$ y Hughes ${ }^{19}$ notamos que las abuelas que cuidan a sus nietos menor cantidad de horas por semana y que tienen un espacio personal respetado, se sienten menos sobrecargadas y más activas y saludables.

En general, lo observado respecto de la participación en las decisiones sobre la crianza de los nietos y la relación con los padres de los nietos es variable y pareciera relacionarse con la personalidad de la abuela. Como en otros trabajos, ${ }^{4}$ la mayoría coincide en que son más flexibles con los nietos y reconoce que es difícil estar tantas horas al cuidado de los niños sin poner límites. Dijeron no sentirse responsables de la crianza y prefieren "cerrar la boca" y no interferir con las reglas paternas.

Las abuelas escogidas para triangular el documento expresaron sentirse identificadas y que el informe refleja lo conversado.

Como limitaciones del trabajo cabe mencionar la muestra acotada de abuelas que seleccionamos, abuelas del área urbana de clase social media, por lo que las conclusiones alcanzan solo a esta población.

Por otro lado, puede discutirse la obtención de la saturación del discurso con solo dos grupos focales. Cabe mencionar que resultó difícil la convocatoria, en parte porque varias abuelas encontraban difícil hacerse un espacio de tiempo para concurrir a los grupos focales.

\section{CONCLUSIONES}

Las abuelas maternas son las más convocadas para cuidar a los nietos como sostén afectivo, económico y por confianza. Este rol de abuelas cuidadoras es un deber implícito en la familia.

Cuidar nietos constituye un acto gratificante 
y placentero. Observamos diferencia en base al tiempo dispensado: mayor cantidad de horas pareciera relacionarse con sentimientos de cansancio, sobrecarga y agotamiento.

La situación más relacionada con la percepción de falta de salud de las abuelas fue el haber estado involucradas en situaciones familiares conflictivas. La participación en las decisiones sobre la crianza de los nietos y la relación con los padres de los nietos es variable y parece relacionarse con la personalidad de las abuelas. Sin embargo, consideramos la necesidad de confirmar estas observaciones con otros estudios.

El cuidado de los nietos proporciona satisfacción, sensación de vitalidad y dinamismo y, como aspectos negativos puede generar estrés, falta de tiempo personal, trastornos en la salud y conflicto con los padres.

\section{BIBLIOGRAFÍA}

1. Szinovacz ME. Grandparents today: a demographic profile. Gerontologist 1998; 38(1): 37-52.

2. Instituto de Mayores y Servicios Sociales-Centro de Investigaciones Sociológicas. Encuesta de condiciones de vida de las personas mayores. Madrid, 2006.

3. Pérez Ortiz L. Las mujeres como recurso de conciliación entre la vida familiar y laboral. Presente y futuro. Madrid: Ministerio de Trabajo y Asuntos Sociales. Instituto de la mujer, Rosa M. Peris, 2000.

4. Osuma MJ. Relaciones familiares en la vejez: vínculos de los abuelos y de las abuelas con los nietos y nietas en la infancia. Rev Mult Gerontol 2006; 16(1): 16-25.

5. Yuni J, Urbano C. Envejecimiento y Género: Perspectivas teóricas y aproximaciones al envejecimiento femenino. Rev Argent Sociol 2008; 10(6): 151-69.

6. Partida R. Trabajadoras de la electrónica en Jalisco: Las abuelas como proveedoras de cuidado infantil. El Cotidiano 2004; 19: 68-77.

7. Minkler M, Fuller-Thomson E. Physical and mental health status of American grandparents providing extensive child care to their grandchildren. J Am Med Womens Assoc 2001; 56(4): 199-205.

8. Minkler M, Fuller-Thomson E, Miller E, Driver D. Depression in grandparents raising grandchildren: results of a national longitudinal study. Arch Fam Med 1997; 6(5): 445-52.

9. Blustein J, Chan S, Guanais FC. Elevated depressive symptoms among caregiving grandparents. Health Serv Res 2004; 39(6 pt 1): 1671-89.

10. Musil C, Ahmad M. Health of grandmothers: a comparison by caregiver status. J Aging Health 2002; 14(1): 96-121.

11. Grinstead L, Leder S, Jensen S, Bond L. Review of research on the health of caregiving grandparents. J Adv Nurs 2003; 44(3): 318-26.
12. LeeS, Colditz G, Berkman L, Kawachi I. Caregiving to children and grandchildren and risk of coronary heart disease in women. Am J Public Health 2003; 93(11):1939-44.

13. Morales AG. El síndrome de la abuela esclava: Pandemia del siglo XXI. Granada: Grupo Editorial Universitario; 2001.

14. Musil CM, Gordon NL, Warner CB, Zauszniewski JA, et al. Grandmothers and caregiving to grandchildren: continuity, change, and outcomes over 24 months. Gerontologist 2011;51(1):86-100.

15. Musil CM. Health, stress, coping, and social support in grandmother caregivers. Health Care Women Int 1998; 19(5):441-55.

16. Ahmad M, Musil CM. Health of grandmothers: a comparison by caregiver status. J Aging Health 2002 14(1): 96121.

17. Balukonis J,Melkus GD, Chyun D. Grandparenthood status and health outcomes in midlife African American women with type 2 diabetes. Ethn Dis 2008;18(2):141-6.

18. Hughes ME, Waite LJ, LaPierre TA, Luo Y. All in the family: the impact of caring for grandchildren on grandparents health. J Gerontol B Psychol Sci Soc Sci 2007; 62(2):S108-19.

19. Kornblit AL, Mendes Diz AM. La salud y la enfermedad: aspectos biológicos y sociales. Buenos Aires: Aique; 2000.

20. Lugones Botell M, Ramírez Bermúdez M. Lo social y lo cultural. Su importancia en la mujer de edad mediana. Rev Cubana Obstet Ginecol 2008;34(1):0-0.

21. Llovet JJ. Servicios de salud y sectores populares: los años del proceso. Buenos Aires: CEDE; 1984.

22. Ktitzinger J. Introducing focus groups. BMJ 1995; 311: 299302.

23. Dick B. Grounded theory: a thumbnail sketch. 2005. [Acceso: 26 enero 2012]. Disponible en: http:/ / www.scu.edu. $\mathrm{au} / \mathrm{schools} / \mathrm{gcm} / \mathrm{ar} / \mathrm{arp} /$ grounded.html.

24. Saltalamacchia H. Del proyecto al análisis: aportes a la investigación cualitativa socialmente útil. Buenos Aires: El Artesano, 2005.

25. Minayo MC. Etapa de análisis o tratamiento del material. En: El desafío del conocimiento. Investigación cualitativa en salud. Buenos Aires: Lugar editorial, 1997. Págs. 165207.

26. Cáceres Mesa M y García Cruz R. Fuentes de rigor en la investigación cualitativa. [Acceso: 2 febrero 2012]. Disponible en: http:/ / www.bibliociencias.cu/gsdl/collect/ index/assoc/HASH0197/eFGFdGbc.dir/doc.pdf.

27. Goodman MR, Rao SP. Grandparents raising grandchildren in a US-México border community. Qual Health Res 2007; 17(8):1117-36.

28. Williamson J, Miller J. Grandmothers raising grandchildren: An exploration of their experiences and emotions. Family Journal 2003; 11: 23-32.

29. Hernández Tezoquipa I, Atenas Monteal ML, Valde Santiso R. El cuidado de la salud en el ámbito doméstico: interacción social y vida cotidiana. Rev Saude Pública 2001; 35(5): 443-50.

30. Musil CM, Warner CB, Sauszniewski JA, Jeanblanc AB, Kercher K. Grandmothers, caregiving, and family functioning. J Gerontol B Psychol Sci Soc Sci 2006; 61(2):S89-98. 